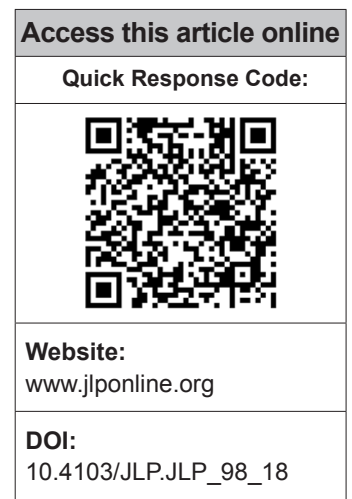

Departments of Microbiology and ${ }^{1}$ Pathology, BLDE (Deemed to be University) Shri B M Patil Medical College, Vijayapura, Karnataka, India

Address for correspondence: Dr. Rashmi Mahesh Karigoudar, Department of Microbiology, Shri B M Patil Medical College, Vijayapura - 586 103,

Karnataka, India.

E-mail: rashmi. karigoudar@bldedu.ac.in

Submission: 24-07-2018 Accepted: 17-12-2018

\title{
Detection of biofilm among uropathogenic Escherichia coli and its correlation with antibiotic resistance pattern
}

\author{
Rashmi M. Karigoudar, Mahesh H. Karigoudar ${ }^{1}$, Sanjay M. Wavare, \\ Smita S. Mangalgi
}

\begin{abstract}
:
BACKGROUND: Escherichia coli accounts for $70 \%-95 \%$ of urinary tract infections (UTIs). UTI is a serious health problem with respect to antibiotic resistance and biofilms formation being the prime cause for the antibiotic resistance. Biofilm can restrict the diffusion of substances and binding of antimicrobials. In this context, the present study is aimed to perform in vitro detection of biofilm formation among E. coli strains isolated from urine and to correlate their susceptibility pattern with biofilm formation.
\end{abstract}

MATERIALS AND METHODS: A total of $100 \mathrm{E}$. coli strains isolated from patients suffering from UTI were included in the study. The identification of $E$. coli was performed by colony morphology, Gram staining, and standard biochemical tests. The detection of biofilm was carried out by Congo Red Agar (CRA) method, tube method (TM), and tissue culture plate (TCP) method. Antimicrobial sensitivity testing was performed by Kirby-Bauer disc diffusion method on Muller-Hinton agar plate. RESULTS: Of the 100 E. coli strains, $49(49 \%)$ and 51 (51\%) were from catheterized and noncatheterized patients, respectively. Biofilm production was positive by CRA, TM, and TCP method were $49(49 \%), 55(55 \%)$, and $69(69 \%)$, respectively. Biofilm producers showed maximum resistance to co-trimoxazole (73.9\%), gentamicin $(94.2 \%)$, and imipenem $(11.6 \%)$ when compared to nonbiofilm producers. Significant association was seen between resistance to antibiotic and biofilm formation with a $P=0.01(<0.05)$.

CONCLUSION: A greater understanding of biofilm detection in E. coli will help in the development of newer and more effective treatment. The detection of biofilm formation and antibiotic susceptibility pattern helps in choosing the correct antibiotic therapy.

Key words:

Antibiotic resistance, biofilm, Escherichia coli

\section{Introduction}

Trinary tract infections (UTIs) are the most common of all bacterial infections. Almost $95 \%$ of UTIs are caused by bacteria. Bacteria follow ascending route of infections in $90 \%$ of UTIs, primarily derived from fecal flora of the host, but hematogenic infections do occur. Escherichia coli accounts for $70 \%-95 \%$ of UTIs. E. coli, an

This is an open access journal, and articles are distributed under the terms of the Creative Commons Attribution-NonCommercial-ShareAlike 4.0 License, which allows others to remix, tweak, and build upon the work non-commercially, as long as appropriate credit is given and the new creations are licensed under the identical terms.

For reprints contact: reprints@medknow.com important member of the normal intestinal microflora in humans and other mammals is a highly versatile bacterium. It may act as harmless gut commensal to intra- or extraintestinal pathogens, being also a frequent colonizer of medical devices and the primary causes of recurrent urogenital infections. ${ }^{[1]}$ Catheterization of the urinary tract increases the risk of bacteriuria in up to $10 \% /$ day. ${ }^{[2]}$ The majority of the patients with an indwelling urinary catheter for 30 days or longer develops bacteriuria. ${ }^{[3]}$

How to cite this article: Karigoudar RM, Karigoudar MH, Wavare SM, Mangalgi SS. Detection of biofilm among uropathogenic Escherichia coli and its correlation with antibiotic resistance pattern. J Lab Physicians 2019;11:17-22. 
UTI is a serious health problem with respect to antibiotic resistance and biofilms formation being the prime cause for the antibiotic resistance. E. coli form intracellular bacterial communities with biofilm-like properties within the bladder epithelium. ${ }^{[4]}$ Biofilm producers exhibit an altered phenotype with respect to growth rate and gene transcription. ${ }^{[5]}$ Biofilm can restrict the diffusion of substances and binding of antimicrobials. This will provide effective resistance for biofilm cells against large molecules such as antimicrobial proteins lysozyme and complement. According to the National Institutes of Health, $\geq 60 \%$ of all microbial infections are caused by biofilms. ${ }^{[4]}$

Biofilm production in E. coli promotes the colonization and leads to increase rate of UTIs, and such infections may be difficult to treat as they exhibit multidrug resistance (MDR). The prevalence of biofilm among uropathogenic E. coli (UPEC) ranges from $60 \%$ to $70 \%{ }^{[4,6,7]}$ Biofilm formation may result in the increased ability of strain causing prostatitis to recurrent UTI. Relapse by UPEC has been related to the ability of pathogenic strains to form biofilm. Several studies observed that $50 \%-70 \%$ isolates collected from patient with relapse infections to be biofilm producer. ${ }^{[8]}$

Fluoroquinolones, cephalosporins, and aminoglycosides can reduce the amount of biofilm produced by E. coli for UTI. Cephalothin, ceftriaxone, ceftazidime, and ciprofloxacin reduce biofilm biomass in susceptible strains. Biofilm in resistance strains significantly decrease with higher concentration of ceftazidime, while ceftriaxone and ciprofloxacin lowered biofilm biomass in resistant strains. Cephalothin does not decrease biofilm in resistant strains. Amikacin reduced the biofilm mass in susceptible and nonsusceptible strains. Ampicillin had no significant effect on biofilm. ${ }^{[9]}$

In modern clinical microbiology, the establishment of bacterial biofilms is considered as pathogenicity trait. Antibiotic resistance of urinary tract pathogens has been known to increase worldwide. ${ }^{[4]}$ Microorganisms growing in a biofilm are intrinsically more resistant to antimicrobial agents than planktonic cells. High antimicrobial concentrations are required to inactivate organisms growing in a biofilm, as antibiotic resistance cans increase 1000 fold.${ }^{[10]}$ In this context, the present study is aimed to perform in vitro detection of biofilm formation among E. coli strains from urine cultures by three different methods isolated and to correlate the biofilm production with antibiotic resistance pattern.

\section{Materials and Methods}

The present study was conducted in the Department of Microbiology at BLDE's (Deemed to be University) Shri
BM Patil Medical College Hospital and Research Center, Vijayapura, Karnataka, India. A total of 100 E. coli strains isolated from urine samples suffering from UTI were identified using conventional methods, and tested for biofilm production by three methods and then performed antibiotic susceptibility testing by Kirby-Bauer diffusion method. Ethical clearance certificate obtained from our institution.

\section{Examination of urine}

1. Macroscopic examination: altered color, presence of turbidity, deposit

2. Microscopic examination: urine centrifuged at $2000 \mathrm{rpm}$ for $15 \mathrm{~min}$. The supernatant discarded, and wet preparation of sediment examined under low and high power to observe pus cells, red blood cell, cast and crystals, and epithelial cells

3. Plating of the urine sample by standard loop technique: samples cultured by semi-quantitative method on MacConkey's agar, cystine lactose electrolyte deficient agar medium, and incubated at $37^{\circ} \mathrm{C}$ for $24 \mathrm{~h}$. Urine culture yielding colony counts of $>10^{5}$ organisms $/ \mathrm{ml}$ of a single type along with $>10$ pus cells / HPF of a centrifuged urine sample interpreted as diagnostic of bacteriuria. Bacterial counts of less than this considered insignificant and growth of more than two types of organisms considered as contamination. The identification of E. coli was performed by colony morphology, Gram staining, and standard biochemical tests.

\section{Methods for detection of biofilm \\ Congo red agar method}

Solid medium containing brain heart infusion broth supplemented with 5\% sucrose, and Congo red inoculated and incubated aerobically for $24-48 \mathrm{~h}$ at $37^{\circ} \mathrm{C}$. The positive result indicated by black colonies with a dry crystalline consistency. Weak biofilm producers usually remain pink, though occasional darkening at the centers of colonies observed. A darkening of the colonies with the absence of a dry crystalline colonial morphology indicates an indeterminate result. ${ }^{[11]}$ The experiment performed in triplicates.

\section{Tube method}

Trypticase Soy Broth with $1 \%$ glucose $(10 \mathrm{~mL})$ inoculated with loopful of colonies from overnight culture plates and incubated for $24 \mathrm{~h}$ at $37^{\circ} \mathrm{C}$. Then tubes decanted and washed with phosphate buffer saline (PBS) (pH 7.3) and dried. Dried tubes stained with crystal violet $(0.1 \%)$. Tubes were washed with deionized water to remove excess stain. Tubes then dried in the inverted position and observed for biofilm formation. ${ }^{[12]}$

a. Positive - visible film lining the wall and bottom of the tube

b. Negative - ring formation at the liquid interface. 


\section{Tissue culture plate method}

Isolates from fresh agar plates were inoculated in Trypticase Soy Broth and incubated for $24 \mathrm{~h}$ at $37^{\circ} \mathrm{C}$, then diluted with fresh Trypticase Soya Broth in 1 in 100 dilution. Individual wells of sterile, polystyrene, 96 well-flat bottom tissue culture plate (TCP) wells filled with $0.2 \mathrm{ml}$ aliquots of the diluted cultures and only broth served as control to check sterility and nonspecific binding of media. The TCP was incubated for $18-24 \mathrm{~h}$ at $37^{\circ} \mathrm{C}$. After incubation content of each well was gently removed by tapping the plates. Then wells were washed four times with $0.2 \mathrm{ml}$ of PBS ( $\mathrm{pH} 7.2$ ) to remove free-floating "planktonic" bacteria. Wells were stained with crystal violet $(0.1 \%)$. Excess stain was rinsed off by washing with deionized water, and the plate was kept for drying. ${ }^{[13]}$ If biofilm is formed by organisms, then wells are uniformly stained with crystal violet. Optical density (OD) of stained adherent bacteria was determined with a micro ELISA auto reader at a wavelength of $570 \mathrm{~nm}$ (OD $570 \mathrm{~nm}$ ). Experiment was repeated thrice, and the data then were averaged, and standard deviation was calculated. The mean OD value obtained from media control was deducted from all the test $\mathrm{OD}$ values.

\section{Antibiotic sensitivity testing}

Antibiotic sensitivity testing was performed by Kirby-Bauer disc diffusion method using Clinical LaboratoryStandards Instituteguidelines. ${ }^{[14]}$ The following antibiotics were used - ampicillin, co-trimoxazole, nitrofurantoin, ciprofloxacin, amoxicillin-clavulanic acid, norfloxacin, gentamicin, piperacillin with tazobactam, cephalexin, nalidixic acid, amikacin, cefoperazone with sulbactam, ceftazidime with clavulanic acid, and imipenem.

Statistical analysis was done for Chi-square $\left(\chi^{2}\right), P$ value, and correlation coefficient $(r)$ using InStat Software. $P<0.05$ is considered as statistical significance.

\section{Results}

Among the 100 E. coli isolates, 49 (49\%) and 51 (51\%) strains were from catheterized and noncatheterized patients, respectively. Of $100 \mathrm{E}$. coli isolates subjected to biofilm production, $69(69 \%)$ strains were positive for biofilm production. Forty-nine (49\%), 55 (55\%), and $69(69 \%)$ were positive for biofilm productions by Congo Red Agar (CRA), tube method (TM), and TCP method, respectively. Forty-seven (47\%) strains were positive for biofilm production by all the above three methods. TCP method was found sensitive. Biofilm production was seen maximum among catheterized patients.

Among the 49 (49\%) catheterized patients, 44 (89.7\%) and $5(10.3 \%)$ strains were biofilm and nonbiofilm producer by TCP method, respectively. By TM method, $38(77.6 \%)$ and $11(22.4 \%)$ were biofilm and nonbiofilm producers, respectively. CRA showed $38(77.6 \%)$ strains as biofilm and $11(22.4 \%)$ as nonbiofilm producers. Among the noncatheterized patients, TCP method detected $25(49.0 \%)$ as biofilm producers and $26(51 \%)$ as nonbiofilm producers. By TM, 17 (33.3\%) and 34 (66.7\%) were biofilm and nonbiofilm producers, respectively. By CRA, $11(21.6 \%)$ and $40(78.4 \%)$ were biofilm and nonbiofilm producers, respectively. Overall $89.7 \%$ and $49 \%$ of E. coli strains were biofilm producer from catheterized patients and noncatheterized patients. The correlation of catheterization and biofilm production by different methods has been given in Table 1 . We found a significant correlation between biofilm production and cauterization. Out of 100 E. coli strains, maximum number of isolates were from male patients $59(59 \%)$ compared to female patients $41(41 \%)$. The maximum age group affected was 21-30 years among female $(17 \%)$ and $51-60$ years among male (17\%). Both among the male and female, the maximum age group affected was $21-30$ years (24\%) followed by 51-60 years (21\%) [Figure 1].

Overall resistance pattern of E. coli strains is shown in Table 2. Among the biofilm producers, maximum resistance was seen to amoxyclav, cephalexin (98.6\%), norfloxacin, ciprofloxacin, gentamicin $(94.2 \%)$, nalidixic acid $(92.7 \%)$, ampicillin $(91.3 \%)$, ofloxacin $(89.9 \%)$, ceftazidime with clavulinic acid (78.3\%), cefoperazone with salbactam $(75.4 \%)$, and co-trimoxozole $(73.9 \%)$. Minimum resistance was seen to imipenem (11.6\%), followed by nitrofurantoin (24.6\%), piperacillin with sulbactam (42\%), amikacin (49.3\%). But among the nonbiofilm producers, maximum resistance was seen to amoxyclav, ampicillin (87.1\%), norfloxacin, cephalexin, ofloxacin $(80.6 \%)$, ceftazidime with clavulinic acid $(77.4 \%)$, ciprofloxacin, nalidixic acid $(74.2 \%)$, cefoperazone with sulbactam $(64.5 \%)$, co-trimoxazole, gentamicin, amikacin, piperacillin with sulbactam $(38.7 \%)$, minimum resistance was seen to imipenem (3.2\%) followed by nitrofurantoin (16\%). The antibiotic resistance among biofilm producing E. coli was found to be higher than that of nonbiofilm with a $P=0.01(<0.05)$ which is statistically significant. The correlation could be established

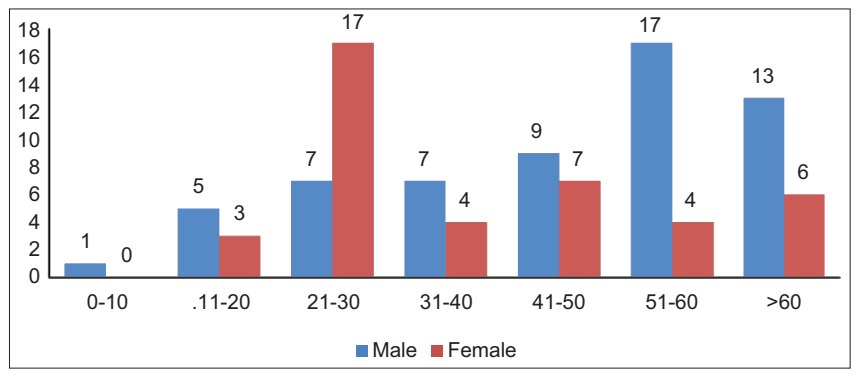

Figure 1: Age-sex wise distribution of Escherichia coli isolates 
Karigoudar, et al.: Biofilm and antibiotic resistance among UPEC

Table 1: Biofilm and nonbiofilm forming Escherichia coli isolates by different methods and its correlation with catheterization/noncatheterization

\begin{tabular}{lcccccc}
\hline Escherichia coli isolates (100) & \multicolumn{2}{c}{ TCP } & \multicolumn{2}{c}{ TM } & \multicolumn{2}{c}{ CRA } \\
\cline { 2 - 6 } & $\begin{array}{c}\text { Biofilm } \\
\text { producers (\%) }\end{array}$ & $\begin{array}{c}\text { Nonbiofilm } \\
\text { producers (\%) }\end{array}$ & $\begin{array}{c}\text { Biofilm } \\
\text { producers (\%) }\end{array}$ & $\begin{array}{c}\text { Nonbiofilm } \\
\text { producers (\%) }\end{array}$ & $\begin{array}{c}\text { Biofilm } \\
\text { producers (\%) }\end{array}$ & $\begin{array}{c}\text { Nonbiofilm } \\
\text { producers (\%) }\end{array}$ \\
\hline Catheterized (49) & $44(89.7)$ & $5(10.3)$ & $38(77.6)$ & $11(22.4)$ & $38(77.6)$ & $11(22.4)$ \\
Noncatheterized (51) & $25(49)$ & $26(51)$ & $17(33.3)$ & $34(66.7)$ & $11(21.6)$ & $40(78.4)$ \\
Total=100 & 69 & 31 & 55 & 45 & 49 & 51 \\
\hline
\end{tabular}

$\chi^{2}=17.57, P=0.0001$ (significant association between cauterization and biofilm production). TCP=Tissue culture plate, TM=Tube method, CRA=Congo Red Agar

Table 2: Antibiotic susceptibility pattern of Escherichia coli isolates

\begin{tabular}{l|c|c}
\hline Antibiotics & Sensitive (\%) & Resistant (\%) \\
\hline Ampicillin & 10 & 90 \\
Co-trimoxazole & 37 & 63 \\
Norfloxacin & 10 & 90 \\
Ciprofloxacin & 12 & 88 \\
Gentamicin & 23 & 77 \\
Nalidixic acid & 13 & 87 \\
Amoxyclav & 5 & 95 \\
Amikacin & 54 & 46 \\
Cephalexin & 7 & 93 \\
Cefoperazone with sulbactam & 28 & 72 \\
Piperacillin/tazobactam & 59 & 41 \\
Ofloxacin & 13 & 87 \\
Imipenem & 91 & 09 \\
Nitrofurantoin & 78 & 22 \\
Ceftazidime/clavulanic acid & 22 & 78 \\
\hline
\end{tabular}

between biofilm production and antibiotic resistance for co-trimoxazole, ciprofloxacin, gentamicin, nalidixic acid, amoxyclav, cephalexin, and imipenem antibiotics, but the correlation could not be found to be significant for rest of the antibiotics [Table 3].

\section{Discussion}

Biofilm producing bacteria are responsible for many recalcitrant infections and are difficult to eradicate. Biofilm production in E. coli promotes colonization and lead to increased UTI. Such infections may be difficult to treat as they exhibit multiple drug resistance.

Saroj et al. showed $69 \%$ isolates as biofilm producers by TM and TCP methods. ${ }^{[6]}$ In a study by Sevanan et al., Congo red method showed $59.4 \%$ strains to be biofilm producer. ${ }^{[4]}$ Significant production of biofilm was seen in $67.5 \%$ isolates of E. coli in a study conducted by Sharma et al. by TCP method. ${ }^{[7]}$ In the present study, biofilm production was seen to be positive in $49 \%, 55 \%$, and $69 \%$ by CRA, TM, and TCP methods, respectively. Our findings are in agreement with the above studies. Whereas in another study reported $72 \%, 100 \%$, and $100 \%$ were biofilm producer by TM, CRA, and TCP methods, respectively. TCP and TM methods did not correlate well with the CRA. ${ }^{[15]}$ Whereas Suman et al. reported a higher rate of biofilm production of $92 \%$ by TCP method. ${ }^{[16]}$
In the present study, majority of biofilm producing E. coli were from catheterized patients (89.5\%). Similarly, Donlan et al. reported in his study, the association of biofilm producing bacteria with urinary catheters. ${ }^{[5]}$ In another study also, biofilm production was more in patients with catheters $(70.3 \%) .^{[16]}$ Saroj et al. categorized the patients into catheterized and noncatheterized (symptomatic and asymptomatic) showed that among 67 catheter-associated UTI, $89.5 \%$ isolates produced biofilm by all the three methods. Among 15 asymptomatic bacteriuria patients, none produced biofilm by TCP method. Among symptomatic UTI, 56\% were biofilm producers by TCP method, $48 \%$ by TM method, and $72 \%$ by CRA method. ${ }^{[13]}$ Similarly in the present study, among the $49(49 \%)$ catheterized patients, $44(89.7 \%)$ and $5(10.3 \%)$ strains were biofilm and nonbiofilm producer by TCP method, respectively. By TM method, 38 (77.6\%) and $11(22.4 \%)$ were biofilm and nonbiofilm producers, respectively. CRA method showed 38 (77.6\%) strains as biofilm and $11(22.4 \%)$ as nonbiofilm producers. Among the noncatheterized patients, TCP method detected $25(49.0 \%)$ as biofilm producers and $26(51 \%)$ as nonbiofilm producers. By TM, 17 (33.3\%) and 34 (66.7\%) were biofilm and nonbiofilm producers, respectively. By CRA, $11(21.6 \%)$ and $40(78.4 \%)$ were biofilm and nonbiofilm producers, respectively.

Mittal et al. reported hemagglutination and gelatinase production of $68 \%$ among biofilm producing UPEC; there was no difference in the production of siderophere in biofilm positive and negative E. coli isolates, greater coprevalance $(78 \%)$ of serum resistance and biofilm formations in UPEC has been found. No statistical significant was obtained between virulence factors and biofilms. ${ }^{[17]}$ When the present study results were compared to those of others, some differences were observed. These variations can be explained by the variation in strain number as well as the assumption that the $E$. coli species tested in some studies were isolated under different environmental conditions and that the nature of the strain was different. On the other hand, it should also be considered that slime production may be affected by various factors, such as medium composition, the presence of carbohydrate, iron and $\mathrm{CO}_{2}$, and oxidation.

InastudyconductedbyPoovendranetal.,allbiofilmforming strains were maximum resistance to amoxyclav (100\%), 
followed by chloramphenicol (100\%), gentamicin and cefotaxime ( $86 \%$ each), ceftazidime $(84 \%)$, cotrimoxazole, and piperacillin with tazobactam (83\% each), and amikacin (70\%). Resistance to co-trimoxazole (83\% vs. $53 \%)$, tetracycline $(75 \%$ vs. $50 \%)$, and ampicillin $(64 \%$ vs. $50 \%$ ) were comparatively higher among biofilm producer than nonbiofilm producer. There was also a significant correlation between biofilm production and resistance to multiple antibiotics. ${ }^{[15]}$ The study conducted by Sevanan et al. showed that biofilm producing organisms are more resistant to antibiotics compared to nonbiofilm producing isolates. The resistant pattern of erythromycin, amikacin, co-trimoxazole, ampicillin, meropenem, chloramphenicol, tobramycin, and gentamicin were found to be in the order of 90.6, 71.9, $65.6,59.3,56.3,56.3 \%, 53.1 \%$, and $50.0 \%$, respectively among biofilm producing isolates. Resistance was seen least with amoxicillin (37.5\%) and cephalexin (18.8\%) ${ }^{[4]}$ In contrast, the present study showed maximum resistance to cephalexin $(98.6 \%)$ among biofilm producers.

In the present study, the correlation between biofilm producer and nonbiofilm producer with antibiotic resistance was found statistically significant with $P=0.01$ for antibiotics: co-trimoxazole, norfloxacin, gentamicin, ciprofloxacin, nalidixic acid, cephalexin, imipenem, and amoxyclav. However, the correlation was not found to be significant $(P=0.92)$ for antibiotics: ampicillin, amikacin, cefoperozone with sulbactam, piperacillin with tazobactam, ofloxacin, nitrofurantoin, and cefatazidime with clavulanic acid among biofilm

Table 3: Comparison of resistance pattern among biofilm and nonbiofilm producers

\begin{tabular}{lcccc}
\hline Antibiotics & \multicolumn{2}{c}{$\begin{array}{c}\text { Biofilm producers } \\
(\boldsymbol{n}=69), \boldsymbol{n}(\%)\end{array}$} & $\begin{array}{c}\text { Nonbiofilm producers } \\
(\boldsymbol{n}=31), \boldsymbol{n}(\%)\end{array}$ \\
\cline { 2 - 5 } & \multicolumn{2}{c}{ Sensitive Resistant } & Sensitive & Resistant \\
\hline Ampicillin & $6(8.7)$ & $63(91.3)$ & $4(12.9)$ & $27(87.1)$ \\
Co-trimoxazole & $18(26.1)$ & $51(73.9)$ & $19(61.3)$ & $12(38.7)$ \\
Norfloxacin & $4(5.8)$ & $65(94.2)$ & $6(19.4)$ & $25(80.6)$ \\
Ciprofloxacin & $4(5.8)$ & $65(94.2)$ & $8(25.8)$ & $23(74.2)$ \\
Gentamicin & $4(5.8)$ & $65(94.2)$ & $19(61.3)$ & $12(38.7)$ \\
Nalidixic acid & $5(8.3)$ & $64(92.7)$ & $8(25.8)$ & $23(74.2)$ \\
Amoxyclav & $1(1.4)$ & $68(98.6)$ & $4(12.9)$ & $27(87.1)$ \\
Amikacin & $35(50.7)$ & $34(49.3)$ & $19(61.3)$ & $12(38.7)$ \\
Cephalexin & $1(1.4)$ & $68(98.6)$ & $6(19.4)$ & $25(80.6)$ \\
Cefoperozone with & $17(24.6)$ & $52(75.4)$ & $11(35.5)$ & $20(64.5)$ \\
sulbactam & & & & \\
Piperacillin/tazobactam & $40(58)$ & $29(42)$ & $19(61.3)$ & $12(38.7)$ \\
Ofloxacin & $7(10.1)$ & $62(89.9)$ & $6(19.4)$ & $25(80.6)$ \\
Imipenem & $61(88.4)$ & $8(11.6)$ & $30(96.8)$ & $1(3.2)$ \\
Nitrofurantoin & $52(75.4)$ & $17(24.6)$ & $26(83.9)$ & $5(16.1)$ \\
Ceftazidime/clavulanic & $15(21.7)$ & $54(78.3)$ & $07(23.6)$ & $24(77.4)$ \\
acid & & & \\
\hline$\chi^{2}=16.79, P=0.01$ (co-trimoxazole, norfloxacin, gentamicin, ciprofloxacin, \\
nalidixic acid, cephalexin, imipenem, amoxyclav), $\chi^{2}=2.036, P=0.92($ ampicillin, \\
amikacin, cefoperozone with sulbactam, piperacillin/tazobactam, ofloxacin, \\
nitrofurantoin, ceftazidime/clavulanic acid) & &
\end{tabular}

and nonbiofilm producers. The similar high rate of resistance among both biofilm and nonbiofilm may be due to other factors associated with antibiotic resistance such as extended-spectrum $\beta$-lactamases (ESBLs), AmpC, beta-lactamases, or carbapenemases. Even though there was no significant correlations for antibiotics such as ampicillin, amikacin, cefoperazone with sulbactam, piperacillin with tazobactam, ofloxacin, nitrofurantoin, and ceftazidime with clavulanic acid with biofilm and nonbiofilm producers, still higher rate of resistance pattern among biofilm producer were observed compared to nonbiofilm producer [Table 3].

When biofilm producing $E$. coli isolates were resistance to two or more classes of antibiotics, were categorized into MDR. Among the MDR pattern of biofilm producing E. coli, there was a significant correlation between biofilm production and resistant to multiple antibiotics with $P=0.001$ [Figure 2]. In the present study, among biofilm producer about $4.3 \%, 8.6 \%$. $14.4 \%, 22.7 \%, 20.2 \%, 17.3 \%$, and $6.0 \%$, and among nonbiofilm producer: $19.3 \%, 6.4 \%$, $19.3 \%, 21.5 \%, 3.2 \%, 3.2 \%$, and $0 \%$, were resistance to multiple drugs of $8,9,10,11,12,13$, and 14 , respectively.

It was observed that there was significant resistance pattern correlation between antibiotics (co-trimoxazole, norfloxacin, gentamicin, ciprofloxacin, nalidixic acid, cephalexin, imipenem, and amoxyclav) and biofilm producers. These antibiotics were not recommended for treating biofilm producing $E$. coli isolates. We recommended piperacillin/tazobactum, nitrofurantoin and imipenem for biofilm producing isolates. We also observed the emergence of resistance against fluoroquinolones, aminoglycosides, co-trimoxazole, ampicillin, and cephalosporins.

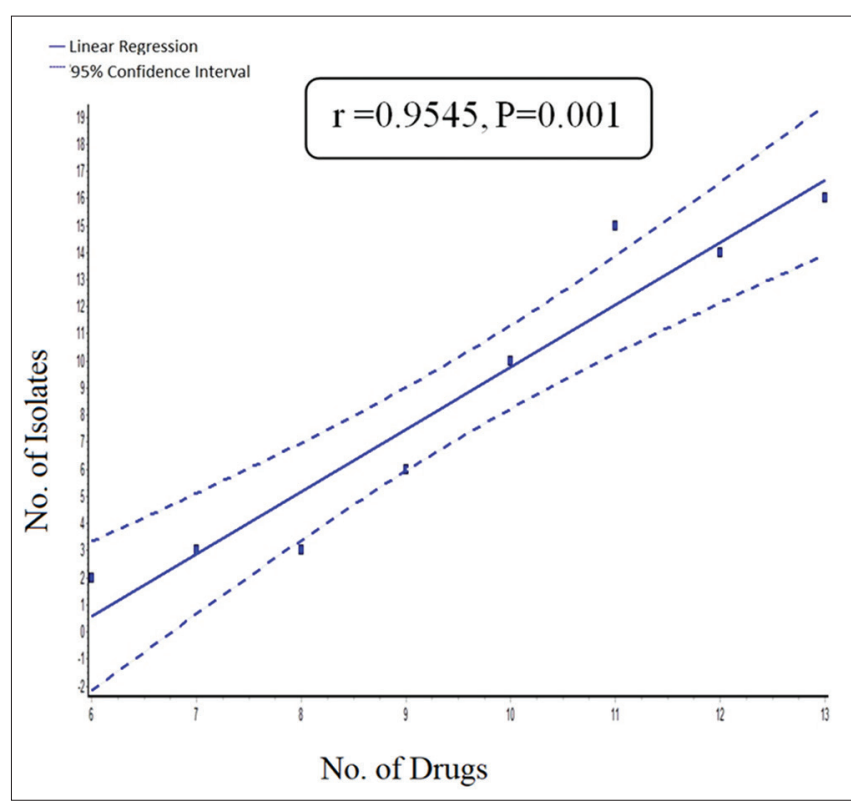

Figure 2: Correlation between biofilm producing isolates and multidrug resistance 
The patients with biofilm producers were resistance to multiple antibiotics as compared to nonbiofilm producers. The patients with biofilm producers need more concentration of drugs as they have to reach the base of biofilm. The common antibiotics used in biofilm producer were nitrofurantoin, piperacillin with tazobactum, imipenem, and amikacin. The biofilm producers with drug-resistant strains were put on more concentration of drugs (piperacillin with tazobactam and amikacin) for favorable outcome. This helped the clinician to decrease the time of hospital stay. The mean duration of therapy for biofilm producers were longer (7-10 days) as compared to nonbiofilm producers (4-5 days). Among the biofilm producers, there were more relapse and recurrent UTI $(10 \%)$, these patients were recommended higher concentrations of sensitive drugs. Most of these patients were recovered completely $(90 \%)$, few did not recover $(10 \%)$, were put on fosfomycin with good results.

Variability in the antibiotic susceptibility pattern has been observed by various authors, which positively reflects the different protocols and panels of antibiotics being used in different hospitals and differences in the geographical locations from where these isolates have been obtained.

\section{Limitation of the study}

In the present study, we have not studied the virulence factors associated with biofilms such as serum resistance, hemagglutinations, and gelatinase productions, so also ESBLs, AmpC, beta-lactamase, and carbapenemases which are associated with antibiotic resistance.

\section{Conclusion}

More than 100 times, the minimum inhibitory concentration of antibiotics is required to eradicate cells within the biofilm. A greater understanding of the nature of biofilm producing E. coli in UTIs will help in the development of new and more effective treatment. Therefore, there is a need to find out a suitable method for detection of biofilm formation among E. coli. From the present study, we have concluded that TCP method is more qualitative and reliable method to detect biofilm producing E. coli. Biofilm forming microorganisms showed resistance to multiple drugs. The implications of the study are that antimicrobials that have wide Gram-negative coverage are particularly effective against biofilm producing E. coli. These antibiotics may be used in the empirical therapy of UTIs caused by biofilm producing UPEC correlating biofilm formation, and antibiotic susceptibility pattern helps in choosing the correct antibiotic therapy.

\section{Financial support and sponsorship} Nil.

\section{Conflicts of interest}

There are no conflicts of interest.

\section{References}

1. Kaper JB, Nataro JP, Mobley HL. Pathogenic Escherichia coli. Nat Rev Microbiol 2004;2:123-40.

2. Warren JW, Tenney JH, Hoopes JM, Muncie HL, Anthony WC. A prospective microbiologic study of bacteriuria in patients with chronic indwelling urethral catheters. J Infect Dis 1982;146:719-23.

3. Stamm WE. Catheter-associated urinary tract infections: Epidemiology, pathogenesis, and prevention. Am J Med 1991;91:65S-71S.

4. Sevanan M, Pongiya U, Peedikavil NJ. Antimicrobial susceptibility pattern of biofilm producing Escherichia coli of urinary tract infections. Curr Res Bacteriol 2011;4:73-80.

5. Donlan RM, Costerton JW. Biofilms: Survival mechanisms of clinically relevant microorganisms. Clin Microbiol Rev 2002;15:167-93.

6. Saroj G, Vivek H, Sujata KK, Reddy M. Correlation between biofilm formation of uropathogenic Escherichia coli and its antibiotic resistance pattern. J Evol Med Dent Sci 2012;1:166-75.

7. Sharma M, Aparna, Yadav S, Chaudhary U. Biofilm production in uropathogenic Escherichia coli. Indian J Pathol Microbiol 2009;52:294.

8. Soto SM, Smithson A, Horcajada JP, Martinez JA, Mensa JP, Vila J, et al. Implication of biofilm formation in the persistence of urinary tract infection caused by uropathogenic Escherichia coli. Clin Microbiol Infect 2006;12:1034-6.

9. González MJ, Robino L, Iribarnegaray V, Zunino P, Scavone P. Effect of different antibiotics on biofilm produced by uropathogenic Escherichia coli isolated from children with urinary tract infection. Pathog Dis 2017;75:1-9.

10. Stewart PS, Costerton JW. Antibiotic resistance of bacteria in biofilms. Lancet 2001;358:135-8.

11. Panda PS, Chaudhary U, Dube SK. Comparison of four different methods for detection of biofilm formation by uropathogens. Indian J Pathol Microbiol 2016;59:177-9.

12. Rashmi MK, Mahantesh BN. Characterization and antibiotic susceptibility pattern of coagulase negative staphylococci with special reference to methicillin resistance. Int J Curr Microbiol App Sci 2016;5:114-20.

13. Christensen GD, Simpson WA, Younger JJ, Baddour LM, Barrett FF, Melton DM, et al. Adherance of coagulase negative staphylococci to plastic tissue cultures; a qualitative model for the adherence of staphylococci to medical devices. J Clin Microbiol 1985;22:996-1006.

14. Clinical and Laboratory Standards Institute. Performance Standards for Antmicrobial Susceptiblity Testing; Twenty-fourth Informational Supplement. CLSI Document M100-S24. Vol. 33. Wayne, PA, USA: Clinical and Laboratory Standards Institute; 2014.

15. Ponnusamy $\mathrm{P}$, Natarajan $\mathrm{V}$, Sevanan $\mathrm{M}$. In vitro biofilm formation by uropathogenic Escherichia coli and their antimicrobial susceptibility pattern. Asian Pac J Trop Med 2012;5:210-3.

16. Suman E, Jose J, Varghese S, Kotian MS. Study of biofilm production in Escherichia coli causing urinary tract infection. Indian J Med Microbiol 2007;25:305-6.

17. Mittal S, Sharma M, Chaudhary U. Biofilm and multidrug resistance in uropathogenic Escherichia coli. Pathog Glob Health 2015;109:26-9. 